\title{
Observations of the CNV during a simple auditory task
}

\author{
HENRY J. MICHALEWSKI and HAROLD WEINBERG \\ Simon Fraser University, Burnaby, British Columbia, Canada V5A 1 S6
}

\begin{abstract}
Slow brain potentials were recorded from normal male and female subjects during a tone discrimination task presented in the CNV interval. Compared to a control condition, CNV averages derived from monopolar scalp sites $\left(C_{z}, F_{3}, F_{4}, T_{3}\right.$, and $\left.T_{4}\right)$ indicated a substantial increase in the amplitude of the potentials during the tone situation. Other CNV features observed included (1) a decline in the slow wave corresponding with a perceptual judgment about the tones, and (2) asymmetries in the CNV that may have been related to differential hemispheric processing of the tone task. The results are discussed in terms of attention, decision processes, and lateral asymmetries.
\end{abstract}

Ever since Walter and his associates (Walter, Cooper, Aldridge, McCallum, \& Winter, 1964) first described the occurrence of the contingent negative variation (CNV) in man, extensive research has been directed at deciphering the relation and significance of this brain wave to behavior, particularly with respect to the theoretical notions of expectancy and attentional processes (Tecce, 1972). Using dc or long time constants, the CNV recorded from scalp electrodes usually appears as a surface-negative slow potential, the amplitude of which is generally larger over the vertex $\left(C_{z}\right)$ than either frontal, temporal, parietal, or occipital locations (Cohen, 1969). In normal subjects, the CNV is more or less symmetrical between the hemispheres; exceptions, however, can be found if special efforts are made to induce asymmetries, for example, lateral differences in the CNV have been observed over speech areas (Zimmermann \& Knott, 1974) and during the performance of numerical operations (Butler \& Glass, 1974).

The CNV is customarily elicited during the foreperiod of a warned reaction time task. The typical CNV paradigm consists of a signal stimulus $\left(S_{1}\right)$ followed by a second stimulus $\left(S_{2}\right)$ to which the subject must react. The interval between $S_{1}$ and $S_{2}$ stimuli is normally fixed and not more than a few seconds in duration. Since the CNV has been intimately connected with psychological as well as preparatory motor functions, the subject's internal activity during the $S_{1}-S_{2}$ interval is, no doubt, critical to the concomitant development of the slow potential. In the usual paradigm, the $S_{1}-S_{2}$ interval is empty or silent with the subject poised and quietly awaiting

The authors wish to thank Dr. R. F. Koopman and Mr. Howard F. Gabert for the development of computer software. Requests for reprints should be sent to Henry J. Michalewski, Developmental Laboratory, Andrus Gerontology Center, University of Southern California, Los Angeles, California 90007. the paired presentation of the $S_{1}$ and $S_{2}$ stimuli. An early report by McCallum and Walter (1968) demonstrated that extraneous stimuli (e.g., an irregular tone) interjected between trials had the effect of reducing the CNV. Later studies have similarly found a suppression of the CNV while distracting events (e.g., presenting numbers of letters for recall, performing a numerical operation) were introduced either before or during the $S_{1}-S_{2}$ signal sequence (Tecce \& Hamilton, 1973; Tecce \& Scheff, 1969). All of these experimentally induced events were thought to affect the development of the CNV by disrupting subjects from properly attending to $S_{1}$ and anticipating $S_{2}$. In our studies of the CNV, we have been interested in modifying and extending the normal CNV paradigm so that the presentation of intervening stimulation was meaningful to the subjects and, in addition, provided the information necessary for making a perceptual judgment. A form of this procedure has been used by McCallum (Note 1) in a continuous vigilance task. He required subjects to monitor a changing digit display and to respond whenever a sequence of three odd numbers was observed. The findings indicated that negative shifts between digit presentations increased in size as the digit combinations approached the response criterion.

In the experiment described below, a simple tone discrimination task was employed which occupied the CNV interval and provided a flow of information that was actively processed by each of the subjects. Since the tone situation was nonverbal and right hemisphere in nature (see Kimura, 1964; Milner, 1971; Shankweiler, 1966), the lateral distribution of the CNV in relation to differential hemispheric processing was also considered.

\section{METHODS}

\section{Stimuli}

A series of tone sets generated from a dual Marconi oscillator 
were prepared and recorded on audio tape. A tone set or trial consisted of two tone pairs presented in succession. With the exception of the last tone in a trial, each tone was followed immediately by the next without interruption; each of the four tones was 750 milliseconds in duration. For each trial, the differences in frequencies between the first tone pair ranged from 200 to $400 \mathrm{~Hz}$, according to a schedule of random variations. The second tone pair was either the same or different from the first pair; if different, only one tone of the sound pair was different. Presented binaurally, overall tone frequencies were selected from a range of 600 to $6,000 \mathrm{~Hz}$ and were equalized with respect to playback volume. A pulse was added before each tone set in order to automatically trigger the start of each trial. The intertrial interval varied from 18 to $30 \mathrm{sec}$. Solid state circuitry assured the precise sequencing of the trigger pulse, tone switching, and tone durations.

\section{Subjects and Recording Techniques}

Five right-handed male and five right-handed female students from Simon Fraser University participated as paid volunteers. Their ages ranged from 18 to 26 years; none of the subjects reported any hearing deficits. Scalp electrical activity was recorded from monopolar electrodes $(\mathrm{Ag}-\mathrm{AgCl})$ cemented at central $\left(\mathrm{C}_{\mathrm{z}}\right)$, frontal $\left(F_{3}, F_{4}\right)$, and temporal $\left(T_{3}, T_{4}\right)$ locations. The monopolar sites were referenced to linked mastoids. Vertical eye movements were monitored with bipolar electrodes placed above and below the right eye; similarly, lateral movements were monitored with electrodes placed at the external canthus of each eye. Electrode impedances were reduced to $3.0 \mathrm{~K} \Omega$ by tapped skin abrasion. EEG activity was amplified and recorded by an eight-channel Elema-Schönander Mingograph. Amplifier sensitivity was adjusted to $50 \mu \mathrm{v} / \mathrm{cm}$ for active sites; time constants were set at $5.0 \mathrm{sec}$ and upper cutoff filters at $30 \mathrm{~Hz}$. Outputs from the recorder were directed on-line to a Hewlett-Packard 2116B computer $(1,024$ points per channel) and stored on disk.

After electrode preparation, the subjects were placed in an electrically shielded sound-attenuating cubicle. The subjects reclined in a bed adjusted for viewing a closed-circuit TV monitor. Average screen brightness was $0.5 \mathrm{fL}$ and was the primary source of lighting in the cubicle. There was a 15-20-min period before the recording started for the purpose of stabilizing the electrodes and to allow the subjects to accommodate to the experimental environment.

\section{Procedure}

Each subject was presented with two conditions. The standard condition (SC) consisted of a $150-\mathrm{msec} 1,000-\mathrm{Hz}$ tone $\left(\mathrm{S}_{1}\right)$ and was followed in $3.0 \mathrm{sec}$ by clicks $\left(\mathrm{S}_{2}\right)$. The subject's task was to terminate the clicks as quickly as possible with a right-hand buttonpress. If the buttonpress occurred before the clicks sounded, the clicks were automatically inhibited and the subject was reminded about the instructions. Several practice trials were given to familiarize the subject with the mode of stimulus presentation, to insure proper eye fixation (provided by a small dot on the TV monitor), and to adopt a pattern of responding compatible with the recording situation. In the tone analysis condition (TA), the subject was instructed to listen to the prerecorded tone sets described earlier. They were instructed to decide whether the second tone pair of each set was the same or different from the first tone pair by a differential response. When the clicks sounded at the end of each tone set, the subject pressed one of two buttons to indicate his judgment and to terminate the clicks. The two-button box was operated with both hands; response positions with respect to same or different were randomized between subjects. Several practice trials were given to assure that the subject understood the tone task and responded correctly. (Correct or incorrect responses to the tone sets were observed but not recorded.)

For both SC and TA conditions, the subjects listened to stimuli from audio speakers located under the bed. Sound levels measured at the subject's head ranged from 65 to $70 \mathrm{~dB}$; tone sets were played from an Uher recorder, and clicks were generated from a Grass S-4 stimulator at a rate of $15 \mathrm{sec}$. For each condition, 16 trials were collected for each of the subjects. The standard condition was administered first. Trials obviously contaminated by eye movement or EMG activity were rejected from on-line averaging. (Except for practice trials, the number of trials rejected never exceeded $14 \%$ of the total for any one subject.) For baseline purposes, data collection was started $100 \mathrm{msec}$ before stimulus presentation; the total sweep duration was $3.5 \mathrm{sec}$. For both SC and TA, the intertrial interval ranged from 18 to $30 \mathrm{sec}$.

\section{Data Treatment}

An amplitude analysis of the plotted data was carried out by dividing each CNV average into four segments corresponding to the four tone periods. In TA, vertical measurements were made from the baseline (determined from the average voltage in the prestimulation period) to a point on the slow wave $50 \mathrm{msec}$ prior to the offset of each tone in the four-tone sequence. The same temporal bounds were also used in measuring CNV amplitudes in SC. The relation between amplitude measures and tone presentations is shown in Figure 1. The obtained values were evaluated in a four-factor analysis of variance design (Sex by Conditions by Electrodes by Time Periods) with repeated measures on the last three factors. Posttest comparisons between the means were carried out with Duncan's multiple-range tests.

\section{RESULTS}

The results for the amplitude measures of CNV activity indicated significant effects for conditions $[\mathrm{F}(1,8)=11.38, \mathrm{p}<.01]$, electrodes $[\mathrm{F}(4,32=19.70$, $\mathrm{p}<.001)$, and time periods $[\mathrm{F}(3,24)=9.91, \mathrm{p}<.001]$. Interactions reaching significant levels included Conditions by Time Periods $[F(3,24)=5.29$, $\mathrm{p}<.01]$, Electrodes by Time Periods $[\mathrm{F}(12,96)=7.93$, $\mathrm{p}<.001$ ], and Conditions by Electrodes by Time Periods $[\mathrm{F}(12,96)=7.89, \mathrm{p}<.001]$. Differences between males and females were not significant $[\mathrm{F}(1,8)<1, \mathrm{p}>.20]$.
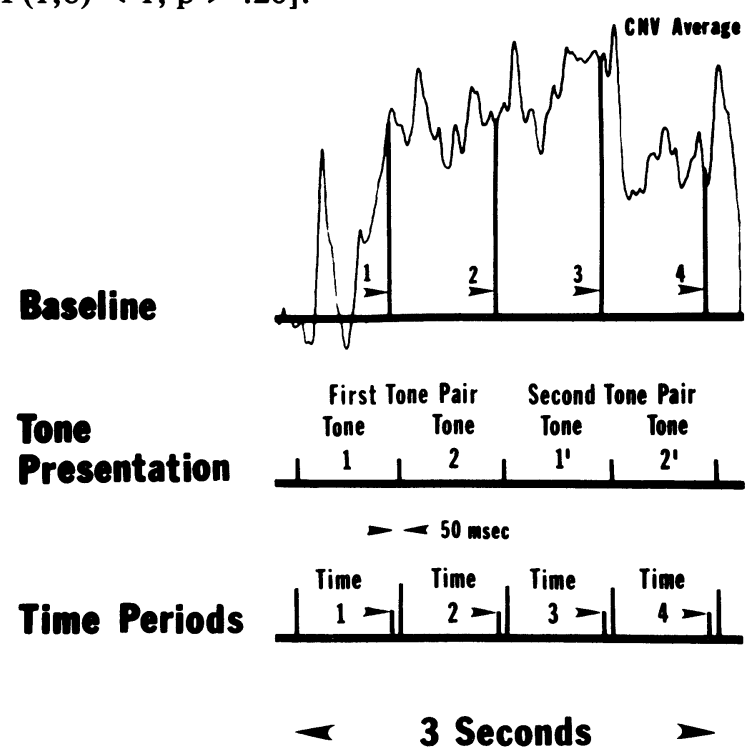

Figure 1. Graphical representation of the relation of the four vertical measures of $\mathrm{CNV}$ amplitude to the corresponding temporal presentation of the tone sequences. For the $\mathrm{CNV}$ average displayed, negative is up. 


\section{Amplitude in $\mu V$}

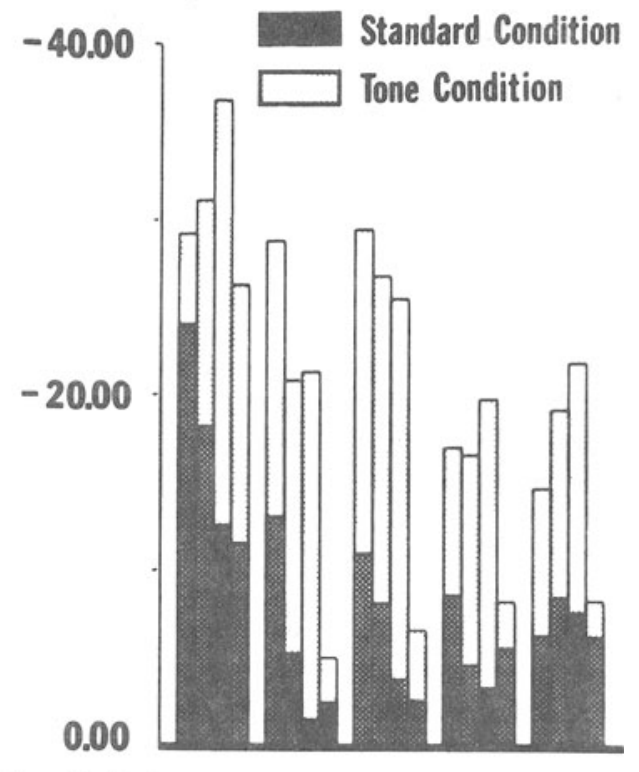

Time Periods $\quad 1-2-3-4 \quad 1-2-3-4 \quad 1-2-3-4 \quad 1-2-3-4 \quad 1-2-3-4$

\section{$\begin{array}{llllll}\text { Electrodes } & \mathrm{C}_{2} & \mathrm{~F}_{3} & \mathrm{~F}_{4} & \mathrm{~F}_{3} & \mathrm{~T}_{4}\end{array}$}

Figure 2. Mean CNV amplitudes at each of the time periods for each electrode site in both the standard and tone-analysis conditions.

\section{Tone Processing}

An overall impression of the various amplitude effects can be seen from the means shown in Figure 2. There was a dramatic increase in the amplitude of the CNV during tone analysis $(20.68 \mu \mathrm{V})$ compared to the standard condition $(8.26 \mu \mathrm{V})$. Overall $\mathrm{CNV}$ activity was larger at the vertex $\left(C_{z}\right)$ than at any of the lateral placements $(p<.001)$; slow-wave activity between frontal and temporal sites was not different. Overall amplitudes at Time Period 1 were greater than at Time $4(\mathrm{p}<.001)$; Times 2, 3, and 4 were not different from each other.

Further analysis of the amplitude data focused upon the triple interaction. Significant and nonsignificant comparisons among amplitudes for conditions and electrodes, which correspond to the differences in Figure 2, are summarized in Table 1. In general, CNV amplitudes in the standard condition tended to decline throughout the CNV interval, whereas in the tone condition amplitudes associated with the first three tones (that is, the two tones of the first pair and the first tone of the second pair) were not significantly changed until the occurrence of the last tone (i.e., final tone of the second pair). Coupled with the fact that most subjects were at least $90 \%$ correct in their judgments about the tone sequences, the decreased CNV for the fourth tone corresponded to the period when the subjects had sufficient information about the preceding tones to make a decision.
Inspection of Figure 2 also showed that the lateral placements appeared to decline more during Time Period 4 than the vertex $\left(C_{z}\right)$ at the same period. However, it could be argued that this drop in the $\mathrm{CNV}$ was due to the long time constants that were used. In order to separate these relative differences quantitatively, proportions of the form Period 4/ (Period 3 + Period 4) were computed for each electrode site in both conditions. Interpretation of the proportions or ratios was as follows: values falling below .5 indicated that CNV amplitudes measured at Time Period 4 were smaller than at Time Period 3; conversely, values above .5 indicated that amplitudes at Time Period 3 were larger than at Time Period 4. An analysis of variance (one-way, within-subjects design) of the proportions in the tone condition confirmed the electrodes effect $[\mathrm{F}(4,36)=$ $13.96, \mathrm{p}<.001]$. Further testing showed that decreases in the CNV were greater for the lateral placements than for the vertex $\left(C_{z}\right)(p<.001)$. A similar analysis of proportions in the standard condition indicated no differences between electrodes $[F(4,36)=1.30, p>.20]$. The ratios for both conditions at each electrode site are shown in Table 2 . Some idea of the actual CNV waveforms which were generated during SC and TA can be seen from the overlaid averages shown in Figure 3. For further comparison purposes, the pooled CNV averages for all subjects in each condition are also included and are shown in Figure 4. In addition to the amplitude differences between the two conditions and the reduced CNV activity of the fourth tone just discussed, evoked responses combined with slow-wave

Table 1

Comparisons Among Time Period Amplitudes in the CNV Interval

\begin{tabular}{|c|c|c|c|c|c|c|}
\hline & & \multicolumn{5}{|c|}{ Electrode Sites } \\
\hline & & $\mathrm{C}_{\mathbf{z}}$ & $\mathrm{F}_{\mathbf{3}}$ & $\mathrm{F}_{\mathbf{4}}$ & $\mathbf{T}_{\mathbf{3}}$ & $\mathrm{T}_{4}$ \\
\hline & & \multicolumn{5}{|c|}{ Time Periods } \\
\hline \multicolumn{2}{|l|}{ Condition } & 1234 & 1234 & 1234 & 1234 & 1234 \\
\hline \multirow{3}{*}{ Standard } & 1 & $* * *$ & $* * *$ & $-* *$ & - * & --- \\
\hline & 2 & $* *$ & -- & -* & -- & -- \\
\hline & 3 & - & - & - & - & - \\
\hline \multirow{3}{*}{ Tone Analysis } & 1 & - $^{*}$ & $* * *$ & - -* & $-{ }^{*}$ & _** \\
\hline & 2 & $*$ & $-*$ & $-*$ & -* & -* \\
\hline & 3 & $*$ & $*$ & $*$ & $*$ & * \\
\hline
\end{tabular}

${ }^{*} p<.001$

Table 2

Mean Proportions Used to Compare CNV Amplitudes at Time Period 4 and Time Period 3

\begin{tabular}{lccccc}
\hline & \multicolumn{5}{c}{ Electrode Sites } \\
\cline { 2 - 6 } Condition & $\mathrm{C}_{\mathbf{z}}$ & $\mathrm{F}_{\mathbf{3}}$ & $\mathrm{F}_{\mathbf{4}}$ & $\mathrm{T}_{\mathbf{3}}$ & $\mathrm{T}_{\mathbf{4}}$ \\
\hline Standard & .45 & .54 & .43 & .58 & .40 \\
Tone Analysis & .41 & .15 & .17 & .27 & .22 \\
\hline
\end{tabular}




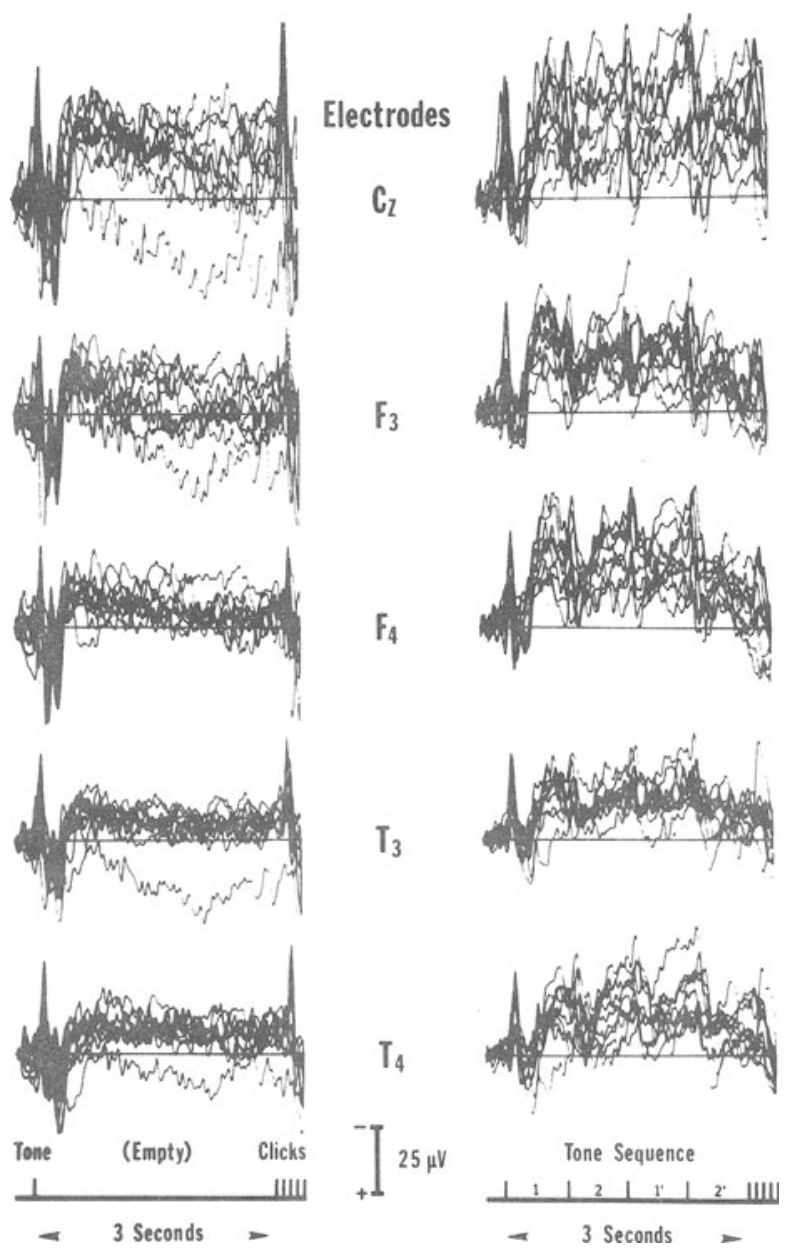

Figure 3. Overlaid CNV averages for each subject at each electrode site in the standard and tone-analysis conditions.

shifts to produce a prominent scalloping effect in the CNV corresponding to the successive presentations of the four tones. This undulating pattern of the slow wave was also very evident in the raw EEG traces. Reaction times to the onset of the clicks were slightly longer in TA (mean $=194.1 \mathrm{msec}$ ) than in $\mathrm{SC}($ mean $=164.9 \mathrm{msec})[\mathrm{t}(9)=2.32, \mathrm{p}<.05]$.

\section{Hemispheric Asymmetries}

A visual inspection of the average CNV potentials between the hemispheres in TA revealed some large asymmetries among many of the subjects. Differences between homologous sites as large as 5 to $12 \mu \mathrm{V}$ were not uncommon. For example, the averages for subject A.K. (male) are shown in Figure 5. Compared to the lateral distribution of the CNV that existed in $\mathrm{SC}$, the records during TA indicated that larger potentials were generated over the right than over the left hemisphere. Conversely, the averages for subject D.M. (male) are shown in Figure 6. When compared to SC, the frontal site on the left hemisphere produced larger CNV potentials in TA than did that on the right hemisphere. In five subjects, larger CNV potentials were generally found over the right than over the left hemisphere, whereas in the remaining subjects potentials either favored the left hemisphere or were close enough in size to preclude a reasonable judgment about differences. Since the asymmetries were not consistent from subject to subject with respect to either the right or left hemisphere, posttest analyses, based on the means from the original analysis of variance, indicated that no lateralization effects in the CNV were present for either the frontal or temporal locations. Similarly, the mandatory right-hand buttonpress of the standard condition did not appear to alter the symmetry of the CNV between the hemispheres.

\section{DISCUSSION}

Compared to the normal slow-potential paradigm, the amplitude findings of the tone condition demonstrated that a perceptual engagement of the subject's attention during the $S_{1}-S_{2}$ interval, and requiring a subsequent judgment response, resulted in an enhancement of the CNV. Since eye movements were
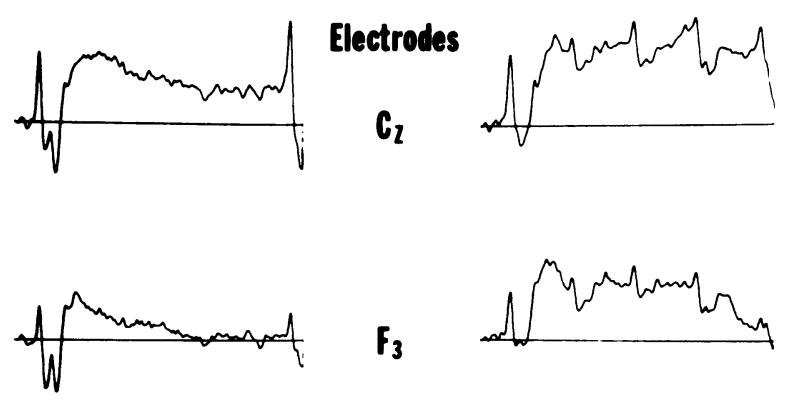

$F_{3}$
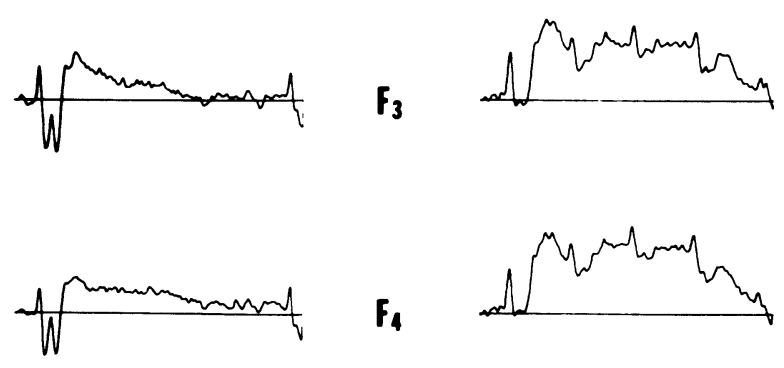

F
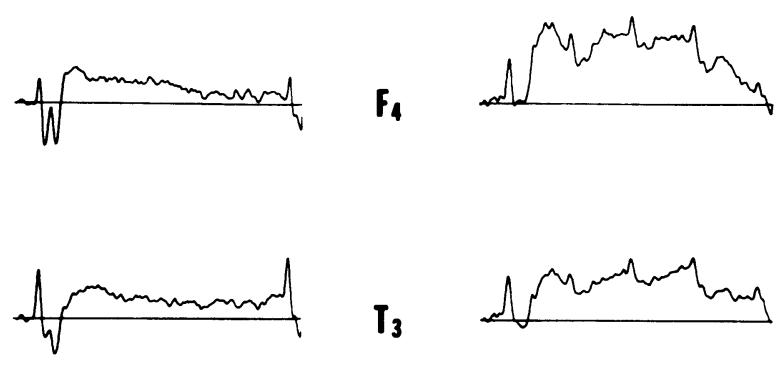

$\mathbf{T}_{3}$
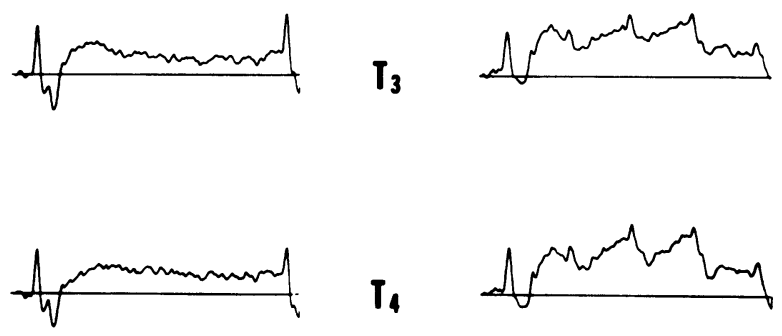

$T_{4}$
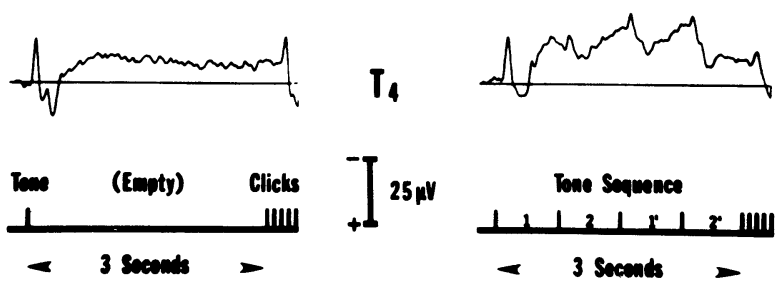

Figure 4. Pooled CNV averages for all subjects in the standard and tone-analysis conditions. 

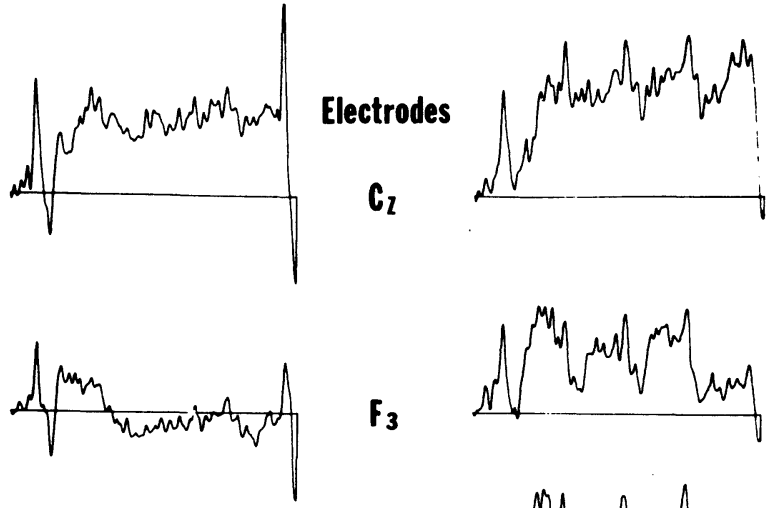

$\mathbf{F}_{3}$
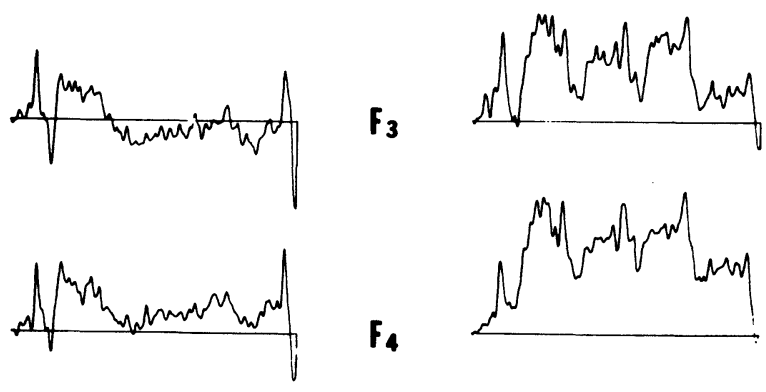

F4
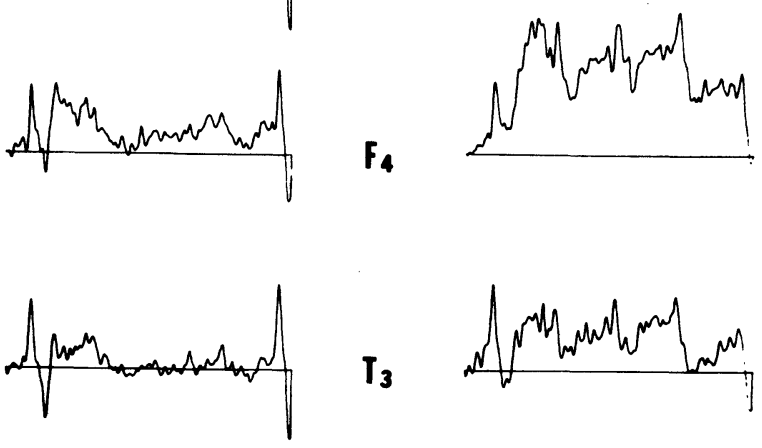

$T_{3}$
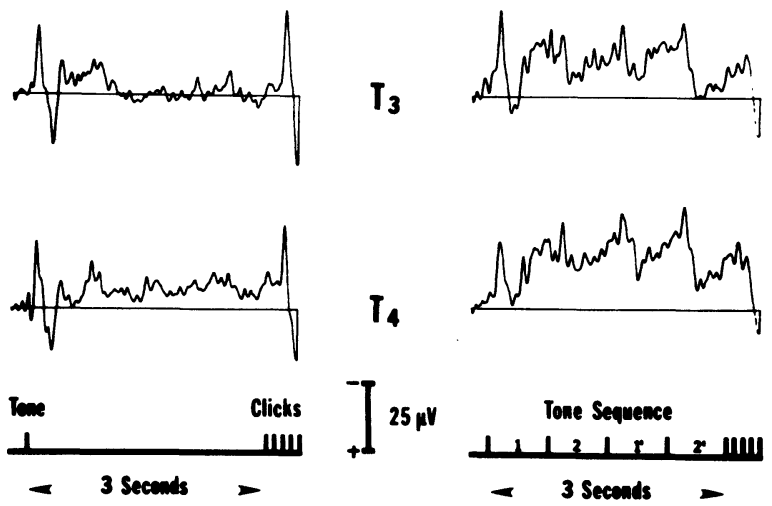

Figure 5. Symmetrical and asymmetrical CNV averages for subject A.K. in the standard and tone-analysis conditions.

carefully monitored, this increase over all scalp locations cannot be attributed to artifacts of ocular origin. Some sort of novelty or motivation effect, however, cannot be ruled out since the subjects were not given the tone sequences in a presentation that did not require active stimulus processing. In distraction experiments, where the subject's attention is divided between the intervening task and attending to $S_{1}$ and responding to $S_{2}, C N V s$ are reduced; the tone condition, on the other hand, replaced the normal signal at $S_{1}$ with a changing input that varied throughout the CNV interval, but which did not have the divisive effects of distraction. We were initially tempted to relate the increased $\mathrm{CNV}$ in the tone condition to some sort of holding or memory process beyond the normal anticipatory contingencies elicited by the CNV paradigm. But the results from an unpublished study by one of us (Michalewski, 1975), in which subjects were tested in the visual modality with graded brightness changes in the CNV interval and which resembled a processing situation analogous to the tone task, did not produce increased
CNVs compared to control conditions. At the moment, therefore, we prefer to explain the amplitude differences between the standard and tone conditions on the basis of a general attention mechanism, affecting substantial portions of the cortical surface and having effects which may be specific to the auditory modality.

The decreased CNV during the presentation of the fourth tone prior to the buttonpress was an unexpected finding. In contrast to the vertex $\left(C_{z}\right)$, proportion or ratio measures at lateral electrode sites provided evidence that positive movement toward baseline might be related to stimulus processing and judgment. When subjects were asked about their solution to the tone task, most said that they had decided upon their response as soon as the fourth tone was heard, even though the design of the experiment allowed, in some cases, sufficient information for a decision as early as the third tone. The reduced or positive-going direction of the CNV is consistent in some respects with interpretations of evoked potential studies in which the positive or P300 component has been related to information processing and the resolution of uncertainty (Sutton, Tueting,
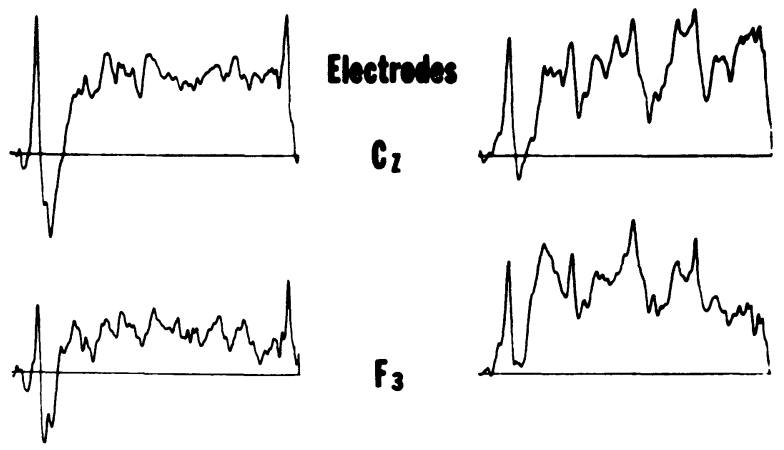

$\mathbf{F}_{3}$

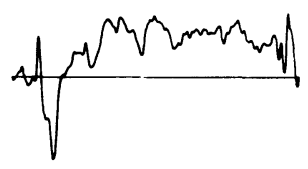

$F_{4}$
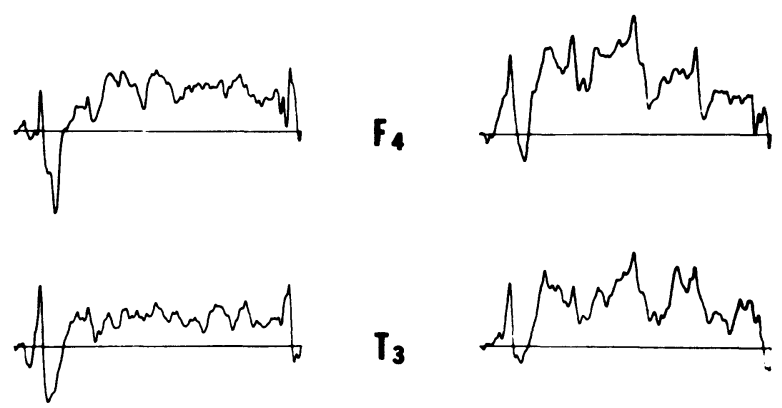

$T_{3}$
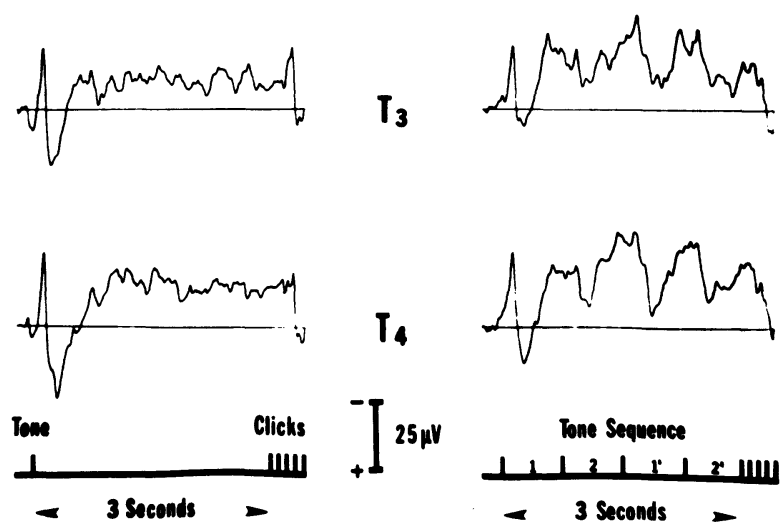

$T_{4}$

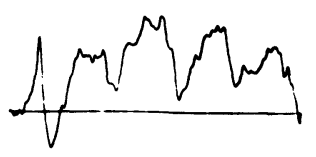

Figure 6. Symmetrical and asymmetrical CNV averages for subject D.M. in the standard and tone-analysis conditions. 
Zubin, \& John, 1967). The slightly longer reaction times (RTs) of the tone condition over the standard suggested an argument against an early decision process during the fourth tone. But this line of reasoning was not pursued since several subjects mentioned some difficulty in perceiving the clicks immediately after the end of the tone sequences, which may have accounted for the longer RTs of the tone condition.

The individual asymmetries which were observed between the hemispheres of many subjects during the tone task merit further investigation, and also serve to generate some interesting speculation as to the underlying origins of the asymmetries. It was assumed that some of the larger differences were not caused by the two-handed operation of the button box because no similar individual asymmetries were observed for the mandatory right-hand buttonpress of the standard condition. Further, in the examples given, the asymmetries can be seen developing long before the subjects could have known which hand might be used for the response. In normal subjects, Zimmermann and Knott (1974) found that CNV potentials preceding simple speech production were larger over the left hemisphere, and the presumed left-hemisphere language system, than over the right hemisphere. CNV asymmetries have also taken a form related to handedness; for example, larger potentials have been observed over the hemispheres contralateral to the preferred hand in a task designed to potentiate hemispheric activity (Butler \& Glass, 1974). If it is assumed that larger CNVs over a particular hemisphere indicate differential engagement or activation, then the asymmetries of the tone condition might be due to an individual dominance for that task, or the differences may be related to underlying hemispheric strategies (see Gazzaniga, 1974) used in the solution of the tonal task. All this, of course, is based on the presumption that the asymmetries, when they occur, are stable and consistent for that individual, and have a direct bearing on the type of information being processed.

\section{REFERENCE NOTE}

1. McCallum, W. C. Brain slow potential changes and motor responses in a vigilance situation. Paper presented at the Third
International Congress on Event-Related Slow Potentials of the Brain, Bristol, August 1973.

\section{REFERENCES}

Butler, S. R., \& Glass, A. Asymmetries in the CNV over left and right hemispheres while subjects await numeric information. Biological Psychology, 1974, 2, 1-16.

Conen, J. Very slow brain potentials relating to expectancy: The CNV. In E. Donchin \& D. B. Lindsley (Eds.), Average evoked potentials (NASA SP191). Washington, D.C: National Aeronautics and Space Administration, 1969.

Gazzaniga, M. Cerebral dominance viewed as a decision system. In S. J. Dimond \& J. G. Beaumont (Eds.), Hemispheric function in the human brain. London: Elek Press, 1974.

KImURA, D. Left-right differences in the perception of melodies. Quarterly Journal of Experimental Psychology, 1964, 14, 355-358.

MCCallum, W. C., \& Walter, W. G. The effects of attention and distraction on the contingent negative variation in normal and neurotic subjects. Electroencephalography and Clinical Neurophysiology, 1968, 25, 319-329.

MICHALEWSKI, H. J. Lateralized cerebral processing and the development of hemispheric slow potentials-the CNV. Unpublished doctoral dissertation, Simon Fraser University, 1975.

MilNeR, B. Interhemispheric differences in the localization of psychological processes. British Medical Bulletin, 1971, 27, 272-277.

ShANKWEILER, D. Effects of temporal-lobe damage on perception of dichotically presented melodies. Journal of Comparative and Physiological Psychology, 1966, 62, 115-119.

Sutton, S., Tueting, P., Zubin, J., \& John, E. R. Information delivery and the sensory evoked potential. Science, 1967, 155, 1436-1439.

TECCE, J. J. Contingent negative variation (CNV) and psychological processes in man. Psychological Bulletin, 1972, 77, 73-108.

Tecce, J. J., \& Hamilton, B. T. CNV reduction by sustained cognitive distraction. In W. C. McCallun \& J. R. Knott (Eds.), Event-related slow potentials of the brain: Their relations to behavior. Amsterdam: Elsevier, 1973.

TECCE, J. J., \& SChEFF, N. M. Attention reduction and suppressed direct-current potentials in the human brain. Science, 1969, 164, 331-333.

Walter, W. G., Cooper, R., Aldridge, V. J., McCallum, W. C., \& Winter, A. L. Contingent negative variation: An electric sign of sensorimotor association and expectancy in the human brain. Nature, 1964, 203, 380-384.

ZimmermanN, G. N., \& KnotT, J. R. Slow potentials of the brain related to speech processing in normal speakers and stutterers. Electroencephalography and Clinical Neurophysiology, 1974, 37, 599-607.
(Received for publication April 14, 1976; revision accepted August 20, 1976.) 